\title{
INDICADORES DE ADOECIMENTO ANTES E APÓS O INGRESSO DA CRIANÇA NA CRECHE*
}

\author{
INDICATORS OF ILLNESS BEFORE AND AFTER THE CHILD’S ENTRY INTO \\ A DAY NURSERY
}

Maria A. C. Andrade**

Maria M. P. Rodrigues ${ }^{* * *}$

\begin{abstract}
Andrade MAC, Rodrigues MMP. Indicadores de adoecimento antes e após o ingresso da criança na creche. Rev Bras Cresc Desenv Hum 2005; 15(2):13-21.

Resumo: Apesar da existência de diversos trabalhos apresentando resultados controversos ou métodos inconsistentes, a literatura continua afirmando relações de causa e efeito entre creche e adoecimento da criança. Assim, investigou-se a ocorrência de doenças em 109 prontuários médicos de crianças de classe média em função de seu ingresso na creche, através da verificação de mudança(s) no número de consultas e no número de doenças nos primeiros doze meses de frequiência à creche, quando comparados com os dois meses anteriores ao ingresso. A análise estatística foi realizada através do teste de Wilcoxon, considerando significativos os valores $\mathrm{p}$ d•0,05. A análise revelou que, na amostra estudada, não houve aumento de consultas nos doze meses após a entrada na creche, porém houve aumento significativo das queixas no $1^{\circ}, 2^{\circ}$ e $3^{\circ}$ bimestres. Pode-se supor que a família e, principalmente, a mãe, quando diante do profissional, com suas concepções baseadas nos numerosos estudos científicos da relação creche/doença, apresenta mais queixas com relação à saúde da criança. Tais queixas, transformadas em diagnóstico de doença levariam à confirmação da predição médica. Contudo, para que se possa avaliar o real efeito dessa profecia auto-realizadora são necessários estudos complementares para testar essa hipótese.
\end{abstract}

Palavras-chave: Adaptação à creche. Saúde da criança. Creche.

\section{INTRODUÇÃO}

Nas últimas décadas, a inserção crescente das mulheres com filhos no mercado de trabalho, a migração em larga escala de populações rurais para centros urbanos industrializados, a diminuição no tamanho das famílias, a quebra na rede de apoio familiar e de vizinhança e um distanciamento físico e psicológico entre os di- ferentes membros da família, praticamente obrigaram a família a buscar soluções alternativas de cuidado infantil ${ }^{1}$. Nos últimos anos, a creche, dentre as alternativas para o cuidado de bebês e crianças pequenas, tem sido adotada, especialmente nos centros urbanos ${ }^{2}$.

O ingresso da criança na creche, principalmente durante o primeiro ano de vida, é um momento crítico, tanto para a criança, quanto

\footnotetext{
Artigo extraído da Dissertação Freqüentar Creche e Adoecer: profecia não concretizada, defendida em 2004 no Programa de Pós-Graduação em Psicologia da Universidade Federal do Espírito Santo.

** Mestre em Psicologia - Programa de Pós-Graduação em Psicologia/UFES geliandrade@ hotmail.com.br Médica pediatra - Instituto Estadual de Saúde Pública (IESP) mariampr@ terra.com.br

Universidade Federal do Espírito Santo - Programa de Pós-Graduação em Psicologia - Av. Fernando Ferrari s/n Campus Goiabeiras 29060-900 Vitória ES

*** Doutora em Psicologia - IPUSP. Docente do Programa de Pós-Graduação em Psicologia /UFES
} 
para sua família e os profissionais da creche, implicando um processo complexo de adaptação ${ }^{3}$, de duração que pode variar de um mês até seis meses ${ }^{2}$. Durante o período de adaptação, as crianças podem apresentar choro, apatia, resistência à alimentação e ao sono e, até mesmo, sintomas físicos como febre e vômitos e, ainda diarréia, bronquite e alergias ${ }^{2,3}$.

A energia exigida para que a criança se adapte às mudanças que ocorrem em sua vida, provoca um desgaste para o organismo, podendo desencadear sérias doenças. Assim, quanto maior o número de mudanças que a criança tiver que enfrentar num período de doze meses, maior a probabilidade de desgaste do organismo, aumentando as chances da ocorrência de problemas de saúde 4 .

O campo da saúde tem investigado e enfatizado as doenças relacionadas com a situação de freqüência de crianças à creche. A maior parte do conhecimento produzido sobre o desenvolvimento da criança advém de estudos feitos nas sociedades industrializadas da Europa e dos Estados Unidos, onde predomina a estrutura de família nuclear, constituída basicamente do pai, mãe e de um a três filhos ${ }^{5}$. A criança fica a cargo da mãe, que conta em geral com pouco apoio no cuidado e educação da criança. A separação ou privação da mãe, que ocorre quando a criança está na creche, tem sido considerada a causa de uma série de distúrbios físicos e mentais.

Na literatura, diversos trabalhos comprovam a associação entre creche e efeitos perniciosos à criança e até à sociedade. Várias pesquisas afirmam que crianças, principalmente bebês, que freqüentam instituições tipo creche apresentam maior risco de adoecimento ${ }^{6-14}$. O risco de adoecimentoé comprovado, em outros trabalhos, pelo aumento da frequiência de consultas médicas por criança na creche ${ }^{15}$, do aumento na duração dos episódios e de maior gravidade nos casos de doença ${ }^{16}$, e maior número de hospitalizações ${ }^{17}$. Como decorrência, as creches têm sido conside- radas, por alguns autores, foco de doenças ${ }^{18} \mathrm{e}$ fonte de prejuízos econômicos, tanto pelos custos (tratamentos, exame clínico e laboratorial, medicação, etc.), como pela ausência dos pais ao trabalho para cuidar dos filhos doentes ${ }^{19}$.

Apesar de inúmeros estudos realizados com metodologia cuidadosamente planejada, em uma revisão das pesquisas sobre a influência da creche para o desenvolvimento infantil, afirma-se que:

[...] embora venha aumentando o número de pesquisas sobre creche, seus resultados não se apresentam conclusivos. Isoladamente, as variáveis pesquisadas têm pouco valor explicativo, mas a dificuldade de se controlarem eficientemente todas as variáveis impossibilita estudos mais abrangentes. Além disso, é freqüentemente inviável a formação de grupos equivalentes para que as comparações entre crianças criadas em casa ou em creche se efetuem dentro de certo rigor metodológico ${ }^{20}$.

Num levantamento bibliográfico ${ }^{21}$ realizado no Current Contents (1995-1998), MedLine (1993-1994) e Lilacs (1982-1997), a partir das palavras-chave "creche/ day care center", foram obtidos 665 artigos, organizados em subgrupos, em função das patologias e dos aspectos abordados, e foi selecionado um subgrupo, o das "doenças infecciosas respiratórias", com 34 artigos (5,1\% dos artigos). Neste estudo, os resultados desses trabalhos mostram-se bastante contraditórios em relação a um conjunto de variáveis, como: raça, tabagismo passivo, condições socioeconômicas e de habitação, número de pessoas e compartilhamento do quarto da criança e presença de outras crianças menores de seis anos como fator de risco para infecções respiratórias. A associação positiva entre creche e infecção respiratória foi atribuída às características da creche (tamanho, número de crianças etc.) e ao número de horas passadas nesse local ${ }^{21}$.

Por outro lado, alguns estudos mostram 
resultado inverso: quanto mais tempo a criança permanece na creche, menor o número de infecções mais tarde, na vida escolar, havendo, na verdade, uma mudança no pico de aparecimento de doenças. Além disso, alguns autores verificaram diminuição na predisposição de asma. Nessa perspectiva, as autoras dessa revisão concluem que houve a utilização de critérios inadequados e, apesar de vários estudos verificarem que o risco relativo de se adquirir infecção respiratória é maior nas crianças que frequientam creche, o significado real desse aumento não deveria justificar a contra-indicação da creche ${ }^{21}$.

Além disso, as autoras criticam, nessas pesquisas, a ênfase na doença, em detrimento da saúde e, também, o procedimento de investigação, em que o adoecimento de crianças que freqüentam creche é comparado ao de crianças cuidadas em casa, pelas mães. Essas comparações, além de inadequadas do ponto de vista científico, revelam o viés implícito dos pesquisadores de considerar a condição de cuidado materno no ambiente doméstico como parâmetro $\mathrm{e}$, até mesmo, condição de normalidade ${ }^{21}$. Nessa perspectiva, esta pesquisa descritiva, do tipo antes e depois, teve como objetivo investigar a ocorrência de doenças em 109 prontuários médicos de crianças de classe média em função de seu ingresso na creche, através da verificação de mudança(s) no número de consultas e no número de doenças nos primeiros doze meses de frequiência à creche, quando comparados com os dois meses anteriores.

\section{MÈTODOS}

Foi selecionada uma creche particular localizada no bairro de Jardim Camburi, no município de Vitória, ES, que atende crianças, de zero a seis anos de idade, provenientes de famílias de camada social média.

Apesar do termo creche designar a ins- tituição, geralmente pública, de cuidado infantil para crianças de zero a três anos, no Espírito Santo o termo creche é utilizado na identificação de qualquer instituição de cuidado infantil, seja pública ou particular, para crianças de zero a seis anos. Por isso, neste trabalho, optou-se pela utilização do termo creche.

Essa creche foi inaugurada em julho de 1997 e conta com uma capacidade para atender 86 crianças de zero a seis anos de idade, provenientes de famílias de camada social média, distribuídas em oito módulos: Sementinha 1 (máximo de 6 crianças, de 3 meses a um ano), Sementinhall (máximo de 8 crianças, de um ano a um ano e meio), Brotinho 1 (máximo de 12 crianças, de um ano e meio a dois anos), Brotinho 11 (máximo de 12 crianças, de 2 a 3 anos), Grupo 1 (máximo de 12 crianças, de 3 a 4 anos), Grupo 11 (máximo de 12 crianças, de 4 a 5 anos), Grupo 111 (máximo de 12 crianças, de 5 a 6 anos) e Grupo IV (máximo de 12 crianças, acima de 6 anos).

A creche contava com um quadro de funcionários, distribuídos nas seguintes funções: uma diretora, um administrador, uma psicóloga, uma pediatra, uma nutricionista, uma cozinheira, uma auxiliar de cozinha, um vigia, duas auxiliares de serviços gerais, duas berçaristas, três auxiliares de creche, oito professoras recreadoras, um professor de música, uma professora de artes, uma professora de inglês e uma secretária.

No ato da matrícula da criança é preenchida pelos pais, geralmente a mãe, uma ficha de anamnese. Das 155 crianças matriculadas até o ano de 2003, foram selecionadas 109 fichas de anamnese, de crianças matriculadas nessa creche, no período de Julho de 1997 a Janeiro de 2003, que frequientaram a creche por um período mínimo de dois meses e que apresentavam a identificação de pelo menos um pediatra assistente.

A escolha, na fase de planejamento da pesquisa, de trabalhar com crianças de camada 
social média deveu-se a uma estratégia para evitar a interferência variáveis relacionadas ao nível socioeconômico na saúde das crianças, além do fato dessas crianças apresentarem acompanhamento pediátrico regular, tendo, consequentemente, registros de sua saúde nos prontuários médicos.

Os pediatras das crianças, identificados nas fichas de anamnese, foram contatados e todos os registros pediátricos das 109 crianças foram disponibilizados para a pesquisa. Apesar de 27 crianças apresentarem mais de um pediatra assistente, todos os 71 pediatras assistentes apontados foram contatados e disponibilizaram os prontuários.

Foram extraídas dos registros pediátricos as consultas, queixas e intercorrências, antes e após o ingresso na creche. Em função de haver crianças com poucos meses de idade, optou-se por fixar como período controle, para todas as crianças, os dois meses anteriores à creche. Foram objeto de análise apenas os prontuários médicos das crianças que tinham registros referentes ao bimestre anterior e ao posterior ao ingresso na creche, o que acarretou a exclusão de 20 prontuários.

São apresentadas a seguir as definições dos quatro indicadores investigados:

Consulta: registro médico da freqüência do paciente ao consultório médico.

Queixa: registro médico do diagnóstico a partir do relato apresentado pelo responsável pela criança, que consistia no motivo principal da consulta, classificado pela Classificação Internacional de Doenças $10^{\mathrm{a}}$ Revisão (CID-10). Os registros decorrentes das consultas de rotina pediátrica (puericultura), de seguimento após o tratamento de uma afecção pediátrica (consulta de revisão) e de avaliação de resultados de exames médicos (consulta para mostrar resultado de exames) não foram incluídos nessa categoria. Apesar de mais de uma queixa familiar ter sido apresentada durante a consulta, apenas a queixa prin- cipal foi considerada, ou seja, no máximo um diagnóstico por consulta.

Intercorrência: registro médico de um ou mais diagnóstico (s) a partir do relato familiar, classificado pela CID-10, ocorridas no intervalo entre a consulta atual e a última consulta.

Doença: Somatório, realizado pela pesquisadora, dos registros médicos de queixas e intercorrências pediátricas, classificadas pela CID-10.

\section{RESULTADOS E DISCUSSÃO}

Os dados brutos, obtidos das fichas de anamnese, foram analisados e interpretados com o auxílio da análise descritiva. Como mostra a Tabela 1, 42,2\% das crianças tinham até 1 ano de idade quando do ingresso na creche, sendo $56 \%$ do sexo feminino. A mediana da idade das crianças ao ingressar na creche era de 1,33. A maior parte eram filhos únicos $(61,5 \%)$, de pais/ mães com nível superior $(90,1 \%$ dos pais e $92,9 \%$ das mães), sendo que $92,6 \%$ das mães trabalhavam fora de casa.

Tabela 1 - Características das crianças estudadas por idade e sexo

\begin{tabular}{cccccccc}
\hline Idades & Meninas & $\%$ & Meninos & $\%$ & Total & $\%$ \\
\hline $0 ?$ & 1 & 22 & $20 \%$ & 24 & $22 \%$ & 46 & 42,2 \\
$1 ?$ & 2 & 19 & $17 \%$ & 13 & $12 \%$ & 32 & 29,4 \\
$2 ?$ & 3 & 12 & $11 \%$ & 8 & $7 \%$ & 20 & 18,3 \\
$3 ?$ & 4 & 4 & $4 \%$ & 2 & $2 \%$ & 6 & 5,5 \\
$4 ?$ & 5 & 3 & $3 \%$ & 1 & $1 \%$ & 4 & 3,7 \\
$5 ?$ & 6 & 1 & $1 \%$ & 0 & $0 \%$ & 1 & 0,9 \\
Total & 61 & $56 \%$ & 48 & $44 \%$ & 109 & 100,0 \\
\hline
\end{tabular}

O número de consultas, intercorrências, queixas e doenças em cada um dos bimestres subseqüentes ao ingresso na creche foi comparado, para cada criança, com o bimestre imediatamente anterior ao ingresso. Para efeito de controle, foi utilizado o autopareamento: cada criança estudada foi comparada com ela mes- 
ma, antes e após sua entrada na creche.

A análise descritiva dos indicadores Consulta, Queixa, Intercorrência e Doença mostra três resultados possíveis: Aumento, Estável e Diminuição, entre o bimestre anterior e cada um dos bimestres posteriores ao ingresso na creche. As crianças que tiveram o mesmo número de consultas, queixas, intercorrências e doenças antes e depois do ingresso na creche foram incluídas na categoria Estável. As crianças que tiveram diminuição no número de consultas, queixas, intercorrências e doenças depois do ingresso na creche foram incluídas na categoria Diminuição. As crianças que tiveram um número maior de consultas, queixas, intercorrências e doenças após o ingresso na creche foram incluídas na categoria Aumento.

Foi utilizado o teste de comparação de médias de Wilcoxon para a analisar cada bimestre, tomando-se o período anterior ao ingresso na creche como controle, considerando-se significativos os valores de $\mathrm{p} d \bullet 0,05$. O estudo estatístico foi realizado com as amostras desbalanceadas, para um melhor aproveitamento dos dados. Assim, no primeiro bimestre foram analisados 89 prontuários, no segundo, 86 , no terceiro 83 , no quarto e no quinto bimestres, 80 prontuários cada e, no sexto, 78 prontuários. O número de prontuários utilizados na análise diminui do primeiro para o sexto bimestre porque quando não havia qualquer registro nos meses subseqüentes o prontuário foi desconsiderado.

Na Figura 1, que mostra a comparação entre as médias de freqüência de consultas no bimestre anterior e cada bimestre posterior ao ingresso na creche, pode-se observar uma tendência de Aumento nas médias de consultas nos três primeiros bimestres; porém, a partir do $4^{\circ}$ bimestre há uma Diminuição bastante evidente nas médias de consultas.

Apesar de o risco de adoecimento ser confirmado, em alguns trabalhos, pelo aumento da freqüência de consultas médicas por cri-

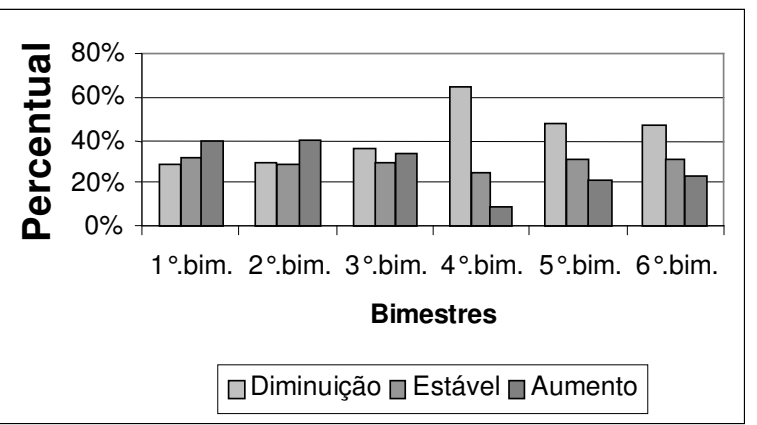

Figura 1 - Comparação entre as médias de frequiência de consultas nos bimestres posteriores com o bimestre anterior ao ingresso na creche.

ança na creche, a análise estatística - teste Wilcoxon - não mostrou mudança significativa no número de consultas após a entrada na creche até o $3^{\circ}$ bimestre, quando ocorreu uma diminuição significativa nas consultas pediátricas nos $4^{\circ}, 5^{\circ}$ e $6^{\circ}$ bimestres $(\mathrm{p}=0,000 ; 0,012$ e 0,018$)$. Assim, provavelmente, a criança não foi levada para consulta pediátrica com maior frequiência após o seu ingresso na creche porque, provavelmente, não adoeceu mais nesse período.

A Figura 2 mostra a comparação entre as médias de freqüência de intercorrências no bimestre anterior e cada bimestre posterior ao ingresso na creche. Observam-se diferenças nas médias das intercorrências, para diminuição, em todos os bimestres, sendo a diferença mais evidente a partir do terceiro bimestre.

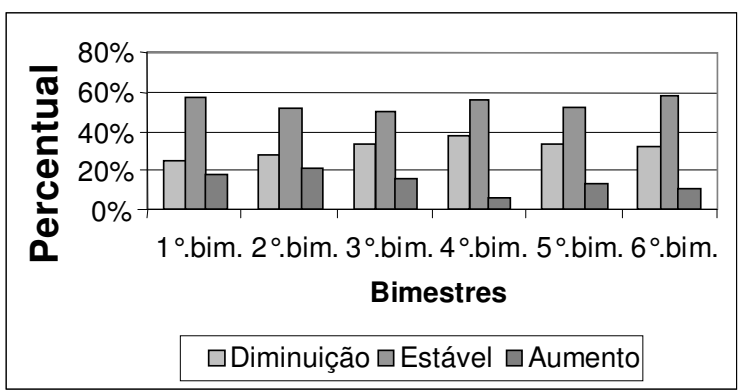

Figura 2 - Comparação entre as médias de freqüência de intercorrências nos bimestres posteriores com o bimestre anterior ao ingresso na creche.

O aumento da incidência de adoecimentos é demonstrado, em algumas pesquisas pelo 
maior número de hospitalizações ${ }^{19}$. A cultura do pronto socorro ${ }^{22} \mathrm{e}$ a grande disponibilidade de unidades particulares de pronto-atendimento infantil na cidade de Vitória faz com que, nesse contexto, o indicador Intercorrência tenha uma grande importância, pelo fato de que, muitas famílias, com crianças em situações de urgência e emergência, procuram um pronto-atendimento pediátrico. Esse atendimento de pronto socorro e a internação hospitalar que pode ter decorrido da consulta são, geralmente, registrados no prontuário médico assistente da criança, como intercorrências, numa próxima consulta. A avaliação das Intercorrências reforça ainda mais o fato de que as crianças não adoeceram mais após a entrada na creche, pelo contrário, houve uma diminuição significativa nas médias das intercorrências no $3^{\circ}, 4^{\circ}, 5^{\circ}$ e $6^{\circ}$ bimestres ( $\mathrm{p}=0,014 ; 0,000$; 0,007 e 0,003 ) após o ingresso na creche.

A Figura 3 mostra a comparação entre as médias de frequiência de queixas no bimestre anterior e cada bimestre posterior ao ingresso na creche. Observa-se aumento nas médias das queixas nos quatro primeiros meses, sendo mais acentuado nos três primeiros.

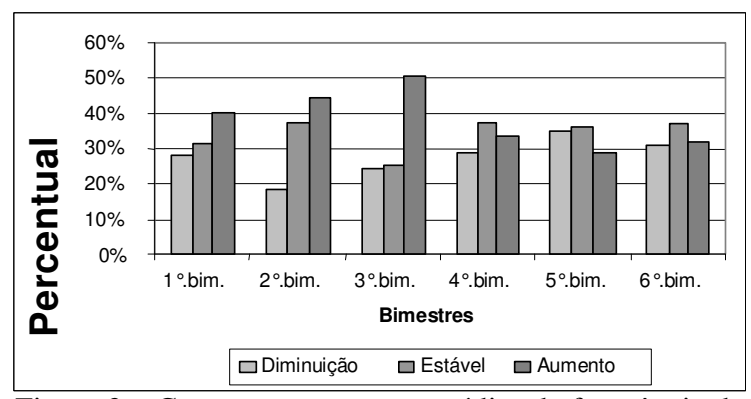

Figura 3 - Comparação entre as médias de frequiência de queixas nos bimestres posteriores com o bimestre anterior ao ingresso na creche.

A maioria das pesquisas citadas comprova o risco de adoecimento pela maior incidência de doenças, maior duração dos episódios e de uma maior gravidade nos casos de doença. Os resultados demonstram que houve aumento das queixas familiares, que são, na consulta médica, classificadas em doenças. Houve, um aumento significativo nas médias de queixas no $1^{\circ}, 2^{\circ}$ e $3^{\circ}$ bimestres ( $p=0,023 ; 0,001$ e 0,008) após o ingresso na creche. Entretanto, o aumento significativo nas queixas trazidas pelos familiares ao consultório pediátrico, que foram traduzidas em doenças pelo médico assistente, ocorreu sem que tenha acontecido um aumento concomitante no número de consultas. Pode-se supor que, apesar de não adoecer mais, e por isso não procurar mais o médico, a família com criança na creche, quando diante do profissional, apresente mais queixas com relação à saúde da criança.

De acordo com a hipótese da profecia autorealizadora é possível que a expectativa de quem faz a predição seja comunicada à outra pessoa, sob formas muito sutis e não intencionais, e, assim, tenha influência em seu comportamento ${ }^{23}$. Dessa forma, diante do profissional de saúde, com suas concepções baseadas nos numerosos estudos científicos da relação creche/doença, mais do que justificados exaustivamente pelocomplexoperíodo de adaptação, a família, e principalmente a mãe, confirmaria a predição: criança na creche adoece mais. Esse processo levaria à confirmação da expectativa criada pela impressão inicial, que é, em grande medida, determinada pelas concepções que se tem da creche. Assim, apesar dessa profecia ter sido confirmada pelo diagnóstico médico a partir da queixa familiar, ela pode realmente não ter se concretizado.

Quanto ao indicador Doença, que é uma junção dos indicadores Queixa e Intercorrência, ocorreu diminuição nas médias de doenças no $1^{\circ}, 4^{\circ}, 5^{\circ}$ e $6^{\circ}$ bimestres, e aumento no $2^{\circ} \mathrm{e}$ $3^{\circ}$ bimestres (Figura 4 ).

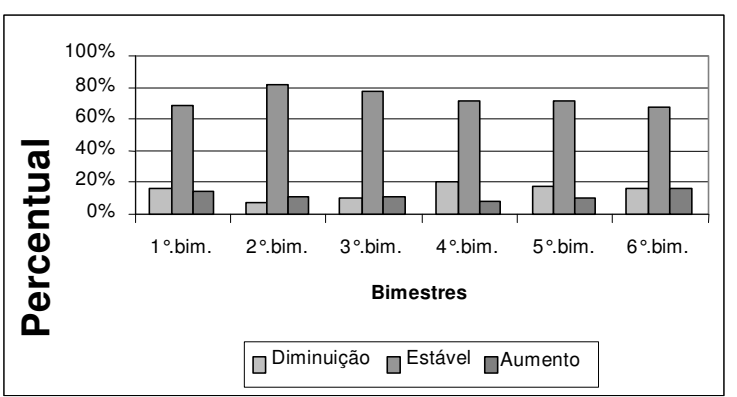

Figura 4 - Comparação entre as médias de freqüência de doenças nos bimestres posteriores com o bimestre anterior ao ingresso na creche. 
O teste de Wilcoxon mostrou que houve uma diminuição significativa nas médias de doenças (a junção de queixas e intercorrências) no $5^{\circ}$ e $6^{\circ}$ bimestres $(p=0,045$ e 0,048$)$ após a entrada na creche. Não houve diferenças significativas nos quatro primeiros bimestres.

\section{CONSIDERAÇÕES FINAIS}

A literatura especializada indica que o período de adaptação da criança na creche envolveria desde o momento de ingresso da criança na creche até o final do primeiro mês, ou ainda, entre três e seis meses após o ingresso. Por outro lado, quanto maior o número de mudanças que a criança tiver que enfrentar num período de 12 meses, maior a probabilidade de desgaste do organismo, aumentando as chances de ocorrência de doenças. Dessa forma, devido aos sintomas decorrentes do processo de adaptação, poder-se-ia esperar um aumento no número de doenças e, conseqüentemente, no número de consultas ao pediatra nesse período. Porém, verificou-se que as consultas e as doenças diminuíram nos bimestres subseqüentes ao ingresso na creche. E, apesar do aumento significativo das queixas no primeiro, segundo e terceiro bimestres após o ingresso na creche, o número de atendimentos pediátricos, incluindo consultas e intercorrências, não aumentou.

A partir dos resultados desta pesquisa não se pode concluir que a creche foi a responsável pela melhoria observada, pois muitos fatores estão envolvidos na saúde da criança. Do mesmo modo, não se pode afirmar que o aumento das queixas, após a entrada na creche, verificado nesta pesquisa, e resultados similares obtidos em outras pesquisas citadas estão diretamente relacionados com a creche. $\mathrm{O}$ adoecimento de crianças, independente de estarem ou não em creches, é complexo e precisa ser melhor analisado.
É importante ressaltar que parece existir, uma cultura social da urgência e emergência. As mudanças ocorridas na nossa sociedade nas últimas décadas culminaram em homens e mulheres sobrecarregados pelo trabalho, ansiosos pela falta de tempo e demandando soluções rápidas para os problemas de saúde de seus filhos. Também, o próprio contexto científico, social e profissional, dominados pelo imediatismo e deslumbramento tecnológico, valorizam a prática médica que promove a resposta rápida, pronta e completa, ou seja, a mais "eficiente" e "especializada" em obter um diagnóstico seguro, através do maior número possível de exames complementares ${ }^{22}$. Após o diagnóstico, a impaciência, característica dos nossos tempos, parece exigir que seja instituído um tratamento imediato, de preferência com drogas de última geração, para aliviar a pressão exercida pela doença da criança na nossa sociedade capitalista e consumidora.

Dentro desse contexto, amplia-se enormemente o campo de conhecimento científico, tanto em extensão quanto em profundidade. Como conseqüência, cada pediatra restringese a uma área de interesse, deixando de ser um pediatra generalista e passando a ser um especialista em fases etárias ou grupos de enfermidade $^{22}$. Assim,

[...] não é de se admirar que, em tal contexto, consultas rotineiras de crianças aparentemente saudáveis, feitas sem grandes recursos tecnológicos e que não trazem, a seus olhos, nenhum 'valor agregado' só sejam feitas quando há tempo disponível - isto é, quase nunca. [...] Acompanhar a evolução de um caso, dando tempo ao tempo para que a condição se esclareça, nem pensar. [...] Se a coisa não progride como desejado, é mais fácil trocar de médico ${ }^{22}$.

Diante de tudo isso, há uma dificuldade do médico em transpor para o coletivo os conceitos e normas da puericultura individual, fazendo com que ele exerça apenas a função 
médico-curativa ${ }^{21}$.

Essa pesquisa traz como contribuição uma reflexão sobre as práticas que estão baseadas e reforçam concepções tradicionais, abrindo um leque de possibilidades à necessidade de novos estudos devido às lacunas ainda existentes. Mais do que associar ou não o adoecimento de crianças à freqüência a uma instituição de cuidado infantil precisamos repensar a ocorrência de doenças no contexto atual, o cuidado das crianças nessa sociedade com homens e mulheres sobrecarregados pelo trabalho e o espaço doméstico vazio. Assim,

[...] uma única consulta não pode permitir o julgamento de uma vida - seja do seu estado de higidez ou de enfermidade. [...] é necessária a compreensão de que a solução imediata de uma crise, ainda que imperiosa, nem sempre, ou quase nunca, encerra o problema. [...] A saúde não repousa sobre hospitais por mais bem fornecidos com a última tecnologia que sejam. [...] Promove-se ou deixase de promovê-la no dia-a-dia humilde da prática ambulatorial ${ }^{22}$.

Na verdade, de acordo com os resultados deste trabalho, a profecia não se concretizou, como mostraram três dos indicadores, mas foi "confirmada" pela percepção daquele que fez a profecia e faz o diagnóstico a partir da queixa familiar. No entanto, a avaliação do real efeito dessa profecia auto-realizadora demanda novos estudos.

\begin{abstract}
In the scientific literature, even the studies that show not only variants but sometimes inconclusive results, do not always manage to free themselves from the relation of cause and effect between nursery education and illness. This study investigated the occurrence of illness in the medical records of 109 middle-class children and the relation of the results to their entry into day nurseries, through the identification of variation(s) in the number of appointments and in the number of illnesses in the first twelve months of attendance at nursery school, when compared with the two months preceding entry. The statistical analysis of these variations was made through the Wilcoxon's test, considering significant the values $\mathrm{p} d \bullet 0,05$. The analysis revealed that, in the samples studied, there was no increase or variation in the variable appointments in the twelve months after entry into nursery education, although there was a significant increase in the complaints in the $1^{\text {st }}, 2^{\text {nd }}$, and $3^{\text {rd }}$ bimesters. It's possible that the family, especially the mother, when faced with a professional influenced by numerous scientific studies showing the relation between nursery education/ illness, presents more health complaints with relation to the child, even though the child does not in fact become ill more frequently, and hence, does not require more frequent medical attention. These complaints, which are transformed into the diagnosis of illnesses, lead to the confirmation of the medical prediction. However, in order to be able to evaluate the real effect of this self-fulfilling prophecy, complementary studies are needed so that this hypothesis can be better evaluated.
\end{abstract}

Key words: Adaptation to the day nursery. Child health. Day nursery.

\section{REFER $\hat{E} N C I A S$}

1. Rossetti-Ferreira MC, Amorim KS, Vitória T. Acre che enquanto contexto possível de desenvolvi mento da criança pequena. Rev Bras Cresc De senv Hum. 1994; 4(2): 35-40.

2. Rapoport A, Piccinini CA. O ingresso e adapta ção de bebês e crianças pequenas à creche: al guns aspectos críticos. Psicol Reflex Crit 2001;
14(1): 81-95.

3. Rapoport A, Piccinini CA. Concepções de edu cadoras sobre a adaptação de bebês à creche. Psic Teor e Pesq 2001; 17(1): 69-78.

4. Lucarelli MDM, Lipp, MEN. Validação do inven tário de sintomas de stress infantil - ISS - I. Psi col Reflex Crit 1999; 12(1): 71-88.

5. Oliveira ZMR, Rossetti-Ferreira MC. Propostas para o atendimento em creches no município de São Paulo: histórico de uma realidade. Cad. Pes 
quisa 1986; 56: 39-65.

6. Barros AJD. Child-care attendance and common morbidity: evidence of association in the litera ture and questions of design. Rev Saúde Pública 1999; 33(1): 98-106.

7. Fonseca W, Kirkwood BR, Barros AJD, Misago C, Correia LL, Flores JAM, et al. Attendance at day care centers increases the risk of childhood pneumonia among the urban poor in Fortaleza, Brasil. Cad Saúde Pública 1996; 12(2): 133-40.

8. Fuchs SC, Maynart RC, Costa LF, Cardoso A, Schierholt R. Duration of day-care attendance and acute respiratory infection. . Cad Saúde Pú blica 1996; 12(3): 291-6.

9. Victora CG, Fuchs SC, Flores JAC, Fonseca W, Kirkwood B. Risk factors for pneumonia among children in a brasilian metropolitan area. Pedia trics 1994; 93(6): 977-85.

10. Holberg CJ, Wright AL, Martinez FD, Morgan WJ, Taussing LM, Group Health Medical Asso ciates. Child day care, smoking by caregivers, and lower respiratory tract illness in the first 3 years of life. Pediatrics 1993; 91(5): 885-92.

11. Blake PA, Ramos S, Mcdonald KL, Rassi V, Go mes TAT, Ivey C, et al. Pathogen-specific risk factors and protective factors for acute diarrheal disease in urban brasilian infants. J Infect Dis 1993; 167: 627-32.

12. Hurwitz ES, Gunn WJ, Pinsky PF, Schonberger LB. Risk of respiratory illness associated with day-care attendance: a nationwide study. Pedia trics 1991; 87(1): 62-9.

13. Fleming DW, Cochi SL, Hightower AW, Broome CV. Childhood upper respiratory tract infections: to what degree is incidence affected by day-care attendance? Pediatrics 1987; 79(1): 55-60.

14. Haskins R, Katch J. Day care and illness: eviden ce cost and public policy. Pediatrics 1986; 77(6): 951-82.

15. Reves RR, Morrow AL, Bartlett III AV, Caruso CJ, Plumb RL, Lu BT, Pickering LK. Child day care increases the risk of clinic visits for acute diar rhea and diarrhea due to rotavirus. Am J Epidemi ol 1993; 137 (1): 97-107.

16. Wald ER, Dashefsky B, Byers C, Guerra N, Taylor F. Frequency and severity of infections in day care. J Pediatr. 1988; 112(4): 540-6.

17. Anderson LJ, Parker RA, Strikas RA, Farrar JA, Gangarosa EJ, Keyserling HL, et al.. Day-care cen ter attendance and hospitalization for lower res piratory tract illness. Pediatrics 1988; 82(3): 300-8.

18. Queiróz DAO, Cardoso DDP, Martelli CMT, Mar tins RMB, Porto SOB, Borges AMT, et al. Risk factors and prevalence of antibodies against he patitis A vírus (HAV) in children from day-care centers, in Goiânia, Brasil. Rev Inst Med Trop 1995; 37(5): 427-33.

19. Amorim K, Yazlle C, Rossetti-Ferreira MC. Binô mios saúde-doença e cuidado-educação em am bientes coletivos de educação da criança peque na. Rev Bras Cresc Desenv Hum 2000;10(2): 03-18.

20. Preuss MRG. Atitudes Maternas e tipo de cuida do alternativo escolhido por mães que trabalham fora. Psic Teor e Pesq 1986; 2(3): 213-24.

21. Amorim KS, Rossetti-Ferreira MC. Análise crítica de investigações sobre doenças infecciosas res piratórias em crianças que freqüentam creche. $\mathbf{J}$ Pediatr 1999; 75(5): 313-20.

22. Machado AL. Atendimento Ambulatorial. Bole tim Informativo da Sociedade Espiritossantense de Pediatria. Sociedade Espiritossantense de Pe diatria 2004; 58. p.1-4.

23. Rosenthal R, Jacobson LF. Pygmalion in the clas sroom. New York: Holt, Rinehart \& Winston; 1968. 